\title{
Oral CPSI-2364 Treatment Prevents Postoperative Ileus in Swine without Impairment of Anastomotic Healing
}

\author{
Tim O. Vilz Nils Sommer $^{\mathrm{a}}$ Philip Kahl ${ }^{\mathrm{b}}$ Dimitrios Pantelis ${ }^{\mathrm{a}} \quad$ Joerg C. Kalffa \\ Sven Wehner ${ }^{\mathrm{a}}$

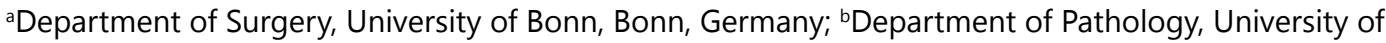 \\ Cologne, Cologne, Germany
}

\section{Key Words}

Postoperative ileus • Muscularis externa $•$ Intestinal inflammation • Anastomotic healing

\begin{abstract}
Background: Postoperative ileus (POI) is an iatrogenic complication of abdominal surgery, mediated by a severe inflammation of the muscularis externa (ME). We demonstrated that orally applicated CPSI-2364 prevents POI in rodents by blockade of p38 MAPK pathway and abrogation of NO production in macrophages. In the present experimental swine study we compared the effect of orally and intravenously administered CPSI-2364 on POI and examined CPSI-2364 effect on anastomotic healing. Methods: CPSI-2364 was administered preoperatively via oral or intravenous route. POI was induced by intestinal manipulation of the small bowel. ME specimens were examined by quantitative PCR for CCL2 chemokine gene expression and myeloperoxidase activity. Functional analyzes included measurement of ileal smooth-muscle ex vivo contractility, in vivo intestinal and colonic transit. Furthermore, anastomotic healing of a rectorectostomy after CPSI-2364 treatment was assessed by perianastomotic hydroxyproline concentration, a histochemically evaluated healing score and anastomotic bursting pressure (ABP). Results: CPSI-2364 abolished inflammation of the ME and improved postoperative smooth muscle contractility and intestinal transit independently of its application route. Hydroxyproline concentration and ABP measurement revealed no wound healing disturbances after oral or intravenous CPSI-2364 treatment whereas histological scoring demonstrated delayed anastomotic healing after intravenous treatment. Conclusion: CPSI-2364 effectively prevents POI in swine independently of its application route. Impairment of anastomotic healing could be observed after intravenous but not oral preoperative CPSI-2364 treatment. Subsumed, an oral preoperative administration of CPSI-2364 appears to be a safe and efficient strategy for prophylaxis of POI.
\end{abstract}

Copyright (c) 2013 S. Karger AG, Basel 


\section{Introduction}

POI is a frequent complication after abdominal surgery [1]. The recovery of gastrointestinal function can last for days with accumulation of fluids and gas, abdominal distension, nausea and vomiting, leading to risk of aspiration and to infectious complications. POI is linked with a high economic burden with costs exceeding US\$ 1 billion per year [2].

Various pathophysiological mechanisms have been investigated and our current knowledge divides POI pathogenesis into an early neurogenic [3] and a late proinflammatory phase[4]. Activation of the latter occurs already during surgery by handling of the gastrointestinal tract (GI-tract) and is a key element in POI development [5, 6]. The inflammation is complexly orchestrated and facilitated by proinflammatory mediator release like IL-6 [7], CCL2 [8] and inhibitory neurotransmitters like nitric oxide (NO) [9]. A hallmark of $\mathrm{POI}$ is the infiltration of circulating monocytes and polymorphonuclear neutrophils (PMN) into the muscularis externa (ME) [5] contributing for manifestation of ME inflammation. In the initial phase, a network of resident ME macrophages was shown to play a key role in POI onset and elimination of these cells protect rodents from POI [10].

Previously we demonstrated that a single preoperative intravenous dose of the tetravalent guanylhydrazone CNI-1493, also known as semapimod, prevents POI in rodents by macrophage-specific inhibition of the p38 mitogen-activated protein kinase (MAPK) pathways and reduction of NO liberation [11]. Macrophage specific inhibition of p38 MAPK pathway and NO release were also shown by others [12, 13]. Unfortunately, intravenous application does not only affect resident ME macrophage function, but also other macrophage populations throughout the whole organism suggesting a possible risk for systemic or distant site adverse effects. In a former study we demonstrated that an oral active mesylate salt of CNI-1493, named CPSI-2364, also prevents POI in rodents [14]. Importantly, oral administration resulted in a nearly exclusive enrichment within the intestinal wall and systemic distribution was not observed. These rodent data firstly revealed orally administered CPSI-2364 as a promising strategy for POI prevention.

It has long been accepted that macrophages facilitate wound healing by supplying growth factors, cytokines and NO and for several mediators crucial roles have been shown in rodent models of anastomotic healing $[15,16]$. As we and others have shown that CNI1493 and CPSI-2364 inhibit macrophage cytokine and NO release, the question arose if preoperative CPSI-2364 treatment for POI prophylaxis could be accompanied by adverse effects of anastomotic wound healing.

In the present study we investigated if orally and intravenously administered CPSI2364 affect postoperative ME inflammation and POI in a standardized swine model of intestinal manipulation. In a subsequent model of rectorectostomy we analyzed CPSI-2364 effects on anastomotic wound healing within the rectum. Together, we provide several lines of evidence that oral preoperative CPSI-2364 application is an effective and safe prophylaxis of POI.

\section{Materials and Methods}

\section{Animals}

All experiments were performed with adult swine (German landrace) of mixed gender. Swine were maintained on a 12 hour light / dark cycle and fed with a rodent diet (ssniff) ad libitum. The animals were fasted for several hours prior to the operation.

All experiments were approved by the committee for animal experiments of North-Rhine Westphalia (LANUV) and performed in accordance with the federal law regarding the protection of animals.

Operation technique and treatment

Swine were fed preoperatively with $1 \mathrm{mg} / \mathrm{kg}$ CPSI-2364 mixed with soft cheese or placebo (soft cheese alone) twice, at $14 \mathrm{~h}$ and $3 \mathrm{~h}$ before the operation. In the intravenous (iv) group, CPSI-2364 resolved in 
Mannitol $1.5 \%$ or placebo (Mannitol $1.5 \%$ ) was administered 60 minutes before laparotomy. The animals were sedated with ketamine, xylazine and atropine $(10,0.2$ and $0.02 \mathrm{mg} / \mathrm{kg}$ respectively) intramuscularly and anesthesia was induced by intravenous application of midazolam $(0.2-0.3 \mathrm{mg} / \mathrm{kg})$, fentanyl $(5 \mu \mathrm{g} /$ $\mathrm{kg}$ ) and pancuronium (0.06 mg/kg). After intubation anesthesia was maintained by isoflurane (1-1.5\%) and oxygen (6 liters/min) inhalation with a ventilation rate of 16 - 18 breaths/min (Sulla 80V, Draeger, Germany). A single preoperative bolus of antibiotics (1 g Mezlocillin) was injected and a central venous catheter was inserted intraoperatively.

\section{Intestinal Manipulation (IM)}

After midline laparotomy the small bowel was eventrated and intestinally manipulated twice from the ligament of Treitz to the ileocecal junction ( $n=6$ for Placebo- or CPSI-treatment, $n=4$ for Sham operated animals). No microvascular bleeding was observed as a result of the IM. For analysis of intestinal transit time we performed a small duodenotomy followed by a placement of 15 radio-opaque glass balls with a diameter of $5 \mathrm{~mm}$ (Worf, Mainz, Germany) in the pars descendens duodeni. For measurement of colonic transit 10 glass balls with a diameter of $7 \mathrm{~mm}$ were put via a small incision into the cecum. Both incisions were closed with Monocryl 4/0 (Johnson and Johnson, Norderstedt, Germany) followed by closure of the abdominal wall using running sutures of Mersilene 0 (Johnson and Johnson, Norderstedt, Germany). Intestinal transit time and colonic transit time were measured simultaneously in the same animal. Postoperative analgesia was performed with Carprofen $(5 \mathrm{mg} / \mathrm{kg}$ ) twice a day. Food and water were provided immediately after the animals had regained mobility after recovery from anesthesia. Sham operated animals were treated in the same way, but were not intestinally manipulated.

Twenty-four hours after closure of the abdominal wall anaesthesia was induced again as described above. $20 \mathrm{~cm}$ of the Ileum (starting $70 \mathrm{~cm}$ orally of the ileocecal valve) were resected and placed in $4^{\circ} \mathrm{C}$ PBS for further investigation. Swine were killed by exsanguination and the entire GI tract was removed and analyzed by X-Ray radiation for examination of intestinal and colonic transit time.

\section{Rectal anastomoses}

Placebo, oral or iv treatment with CPSI-2364 was performed as described above ( $\mathrm{n}=8$ for each group). After laparotomy the middle third of the rectum was transected followed by a rectorectostomy with one layer of continuous suture using 4/0 Monocryl (Johnson and Johnson, Norderstedt, Germany). None of these animals received IM.

For postoperative care, swine were brought to an animal intensive care unit and laboratory parameters were controlled at $6 \mathrm{~h}, 24 \mathrm{~h}$, postoperative 2, postoperative day 4, postoperative day 6 after the operation. Postoperative analgesia was performed with Carprofen $(5 \mathrm{mg} / \mathrm{kg})$ twice a day. Food and water were provided immediately after the animals had regained mobility. Six days after rectorectostomy anesthesia was induced again as described above. The abdominal wall and the anastomotic region were inspected for wound healing disturbances or abscess formation followed by resection of the anastomosis including surrounding tissue. The tissue was placed in $4^{\circ} \mathrm{C}$ PBS for further investigation. Subsequently, Swine were killed using T-61 (Fa. Intervent, Unterschleißheim, Germany) intravenously.

\section{Gene expression analyzes}

Gene expression was analyzed in an isolated ileal ME specimen that was taken $70 \mathrm{~cm}$ orally of the cecum $24 \mathrm{~h}$ after operation ( $\mathrm{n}=6$ for Placebo- or CPSI-treatment, $\mathrm{n}=4$ for Sham operated swine). Total RNA was isolated, reverse transcribed to cDNA and analyzed by quantitative PCR as described before [10]. Primers used for CCL2 amplification were (forward) 5'-ATT CTC CAG TCA CCT GCT CG-3' and (reverse) 5'-TCA AGG CTT CGG AGT TTG G-3'. The PCR reaction was performed in SYBR Green Mastermix with $10 \mathrm{ng}$ cDNA for 40 cycles $\left(95^{\circ} \mathrm{C} 15 \mathrm{sec}, 60^{\circ} \mathrm{C} 1\right.$ minute) on an AbiPrism $7900 \mathrm{HT}$. Data quantification was performed by the $\Delta \Delta$ CT method. All reagents were obtained from Applied Biosystems, Darmstadt, Germany.

\section{Myeloperoxidase (MPO) detection}

An MPO - assay of small bowel ME specimen was performed as described before ( $\mathrm{n}=6$ for Placebo- or CPSI-treatment, $n=4$ for Sham operated swine) [17]. MPO activity was calculated as $\mu \mathrm{U} / \mathrm{mg}$ protein. 


\section{In vitro contractility}

In vitro contractility of circular ME strips (harvested $70 \mathrm{~cm}$ orally of the ileocecal valve) was measured $24 \mathrm{~h}$ after IM as described before $[18,19]$. Briefly, mucosa-free ME strips ( $\mathrm{n}=6$ muscle strips per animal) were equilibrated in a standard Krebs-Henseleit buffer perfused organ chambers at $37^{\circ} \mathrm{C}$ and underwent alternating stimulation with increasing doses of bethanechol (1-100 mol/L) and wash periods with buffer (group size was $n=6$ for placebo- or CPSI-treatment and $n=4$ for Sham-operated swine). The contractile response was recorded and analyzed with ChartPro Software (ADInstruments, Heidelberg, Germany) and calculated as $\mathrm{g} / \mathrm{mm}^{2} / \mathrm{s}$.

\section{Intestinal and colonic transit}

Intestinal and colonic transit were analyzed simultaneously $24 \mathrm{~h}$ after IM by X-Ray detection of intraoperatively administered 5 or $7 \mathrm{~mm}$ radio-opaque glass beads, respectively. The $5 \mathrm{~mm}$ markers were counted in 22 equally sized segments (stomach, 10 small bowel segments, cecum and 10 large bowel segments). Colonic transit was measured by distribution of the $7 \mathrm{~mm}$ beads within in the cecum and 10 large bowel segments. In both transit examinations we investigated $n=6$ animals using Placebo- or CPSItreatment and $n=4$ in the Sham group. The geometric center (GC) was calculated using the following formula: GC $=\Sigma$ (\% of total glass balls per segment * segment number) / 100. The data were expressed as the percentage of glass balls per segment and plotted in a median histogram

\section{Hydroxyproline concentration in anastomotic tissue}

For analysis of hydroxyproline concentration (HPC) a $2 \times 2 \mathrm{~cm}$ measuring piece of anastomotic tissue was chosen from each swine ( $\mathrm{n}=8$ for each group). HPC was quantified as described previously $[20,21]$ and calculated using the following formula: [absorption of sample / coefficient of the standard curve] $\mathrm{x} 666.7=$ $\mu \mathrm{g}$ hydroxyproline / $\mathrm{g}$ bowel.

\section{Histochemical analysis}

Anastomotic segments were dissected 6 days after surgery, fixed in $4 \%$ paraformaldehyde, embedded in paraffin and $5 \mu \mathrm{M}$ sections were stained with hematoxylin-eosin ( $\mathrm{n}=8$ in each group). Microscopical analysis was conducted by a blinded pathologist and anastomotic healing score was calculated using a method modified from Biert et al. [22]. A score from 1 to 5 was assigned for mucosal continuity, muscular continuity, re-epithelisation, and granular tissue formation $(1=$ none, $2=$ poor, $3=$ moderate, $4=$ good, $5=$ excellent and completely). The score was calculated from the sum of the individual scores assigned to each of the parameters assessed.

\section{Anastomotic bursting pressure (ABP)}

$\mathrm{ABP}$ of the rectorectostomy was measured immediately after the animals ( $\mathrm{n}=8$ in each group) were killed. A $15 \mathrm{~cm}$ segment of the colon including the anastomosis was resected and carefully cleared of fecal contamination. The proximal end was ligated twice using a Mersilene 0 suture (Johnson and Johnson, Norderstedt, Germany). An endotracheal tube $(10 \mathrm{CH})$ was inserted intraluminally within the rectum at the distal end and 2 stay sutures (Mersilene 0 ) were tied to prevent leakage. The other end of the tube was connected to an oxygen port in the wall and placed in a water bath. Oxygen was infused at a constant rate of $6 \mathrm{l} / \mathrm{min}$. The intraluminal pressure $(\mathrm{mmHg})$ was measured and recorded using a pressure transducer with an amplifier and analyzed using the Biopac A/D systems (Acknowledge_ Software; Biopac Systems, Santa Barbara, CA). ABP was indicated as a sudden loss of pressure or sudden outlet of bubbles and defined as the maximum intraluminal pressure prior to leakage.

\section{Drugs and Solutions}

PBS was purchased from Lonza (Verviers, Belgium). All other chemicals used for this study were purchased from Sigma Aldrich (Taufkirchen, Germany). CPSI-2364 was kindly provided by Cytokine PharmaSciences, Inc. (King of Prussia, USA).

\section{Data analysis}

Statistical analysis was performed with Prism software (GraphPad, CA, USA) using 1-way ANOVA followed by a Bonferroni's posttest or an unpaired t-test (analysis of smooth muscle contractility). Box and whiskers plots indicate mean \pm SD and the range from minimum to maximum. 
Fig. 1. IM-induced increase in CCL2 gene expression and MPO activity is abolished by CPSI-2364 treatment. (A) Preoperative oral and iv CPSI2364 application resulted in a significant reduction of CCL2 transcripts within the ME compared to the placebo-treated group $24 \mathrm{~h}$ after IM ${ }^{* *} \mathrm{p}<0.01,{ }^{* * *} \mathrm{p}<0.001, \mathrm{n}=4$ for sham, $\mathrm{n}=6-8$ for IM groups). (B) MPO activity within the ME is significantly increased $24 \mathrm{~h}$ after IM in placebotreated but not in orally or intravenously CPSI2364 treated swine $\left({ }^{* *} \mathrm{p}<0.01, \mathrm{n}=4\right.$ for sham, $\mathrm{n}=6$ for IM groups).

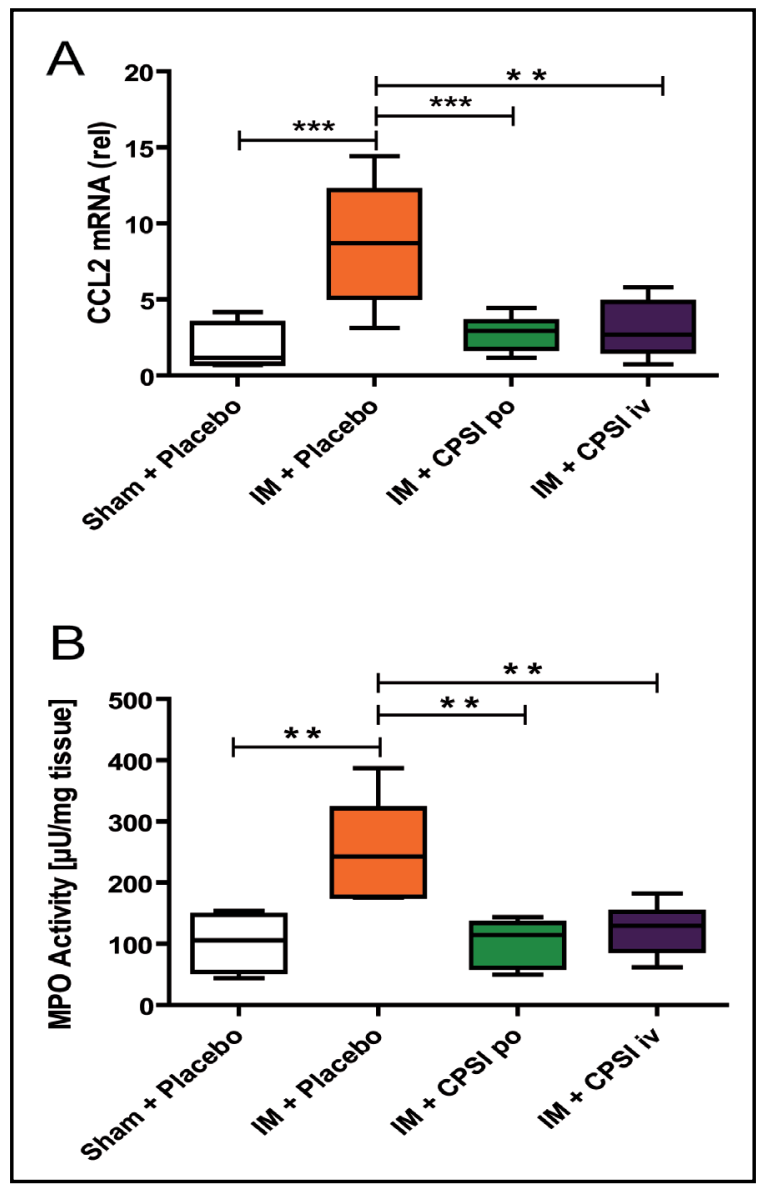

\section{Results}

In the present study we analyzed the influence of CPSI-2364 on the development of POI in a swine model. In a subsequent study CPSI-2364 effects on anastomotic healing were analyzed after partial rectal resection and rectorectostomy.

\section{POI study}

CPSI-2364 ameliorates chemokine transcription in swine ME. As shown in rodent models before, POI is based on extravasation of circulating monocytes in a CCL2 dependent manner [10]. In our swine model, CCL2 transcripts were upregulated in placebo-treated ( 8.8 fold \pm 3.3 ) swine $24 \mathrm{~h}$ after IM. After CPSI-2364 treatment, CCL2 mRNA levels (oral: 2.8 fold \pm 1.1 ; iv: 3.1 fold \pm 1.9 ) were comparable to sham-operated controls $(1.8$ fold \pm 1.6$)$ (Fig. $1 \mathrm{~A})$.

CPSI-2364 reduces PMN infiltration. Next, we investigated whether CPSI-2364 affects IM-induced leukocyte extravasation into small bowel ME by indirect measurement of MPO activity in the ileum. In sham-operated swine, a basal MPO activity of $102.3 \pm 48.6 \mu \mathrm{U} / \mathrm{mg}$ tissue was measured $24 \mathrm{~h}$ after laparotomy (Fig. 1B). Oral $(102.9 \pm 38.3 \mu \mathrm{U} / \mathrm{mg}$ ) or intravenous $(123.8 \pm 41.5 \mu \mathrm{U} / \mathrm{mg})$ CPSI-2364 treatment significantly prevented IM-induced MPO activity increase compared to placebo treated swine $(254.2 \pm 87.7 \mu \mathrm{U} / \mathrm{mg})$.

CPSI-2364 diminishes IM-induced smooth muscle dysfunction. In a set of functional experiments we next measured contractile activity of isolated ileal circular smooth muscle strips in an in vitro organ bath setting and GI motility in vivo. Ileal muscle strips demonstrated an impaired contractility to increasing bethanechol between IM and shamoperated swine after IM. (Fig. 2) ( $1 \mu \mathrm{M}: 0.04 \pm 0.01$ vs. $0.18 \pm 0.07 \mathrm{~g} / \mathrm{mm}^{2} / \mathrm{s} ; 3 \mu \mathrm{M}: 0.15 \pm 0.03$ vs. $0.53 \pm 0.18 \mathrm{~g} / \mathrm{mm}^{2} / \mathrm{s} ; 10 \mu \mathrm{M}: 0.42 \pm 0.09$ vs. $0.92 \pm 0.19 ; 30 \mu \mathrm{M}: 1.1 \pm 0.16$ vs. $2.3 \pm 0.51 ; 100 \mu \mathrm{M}$ : 


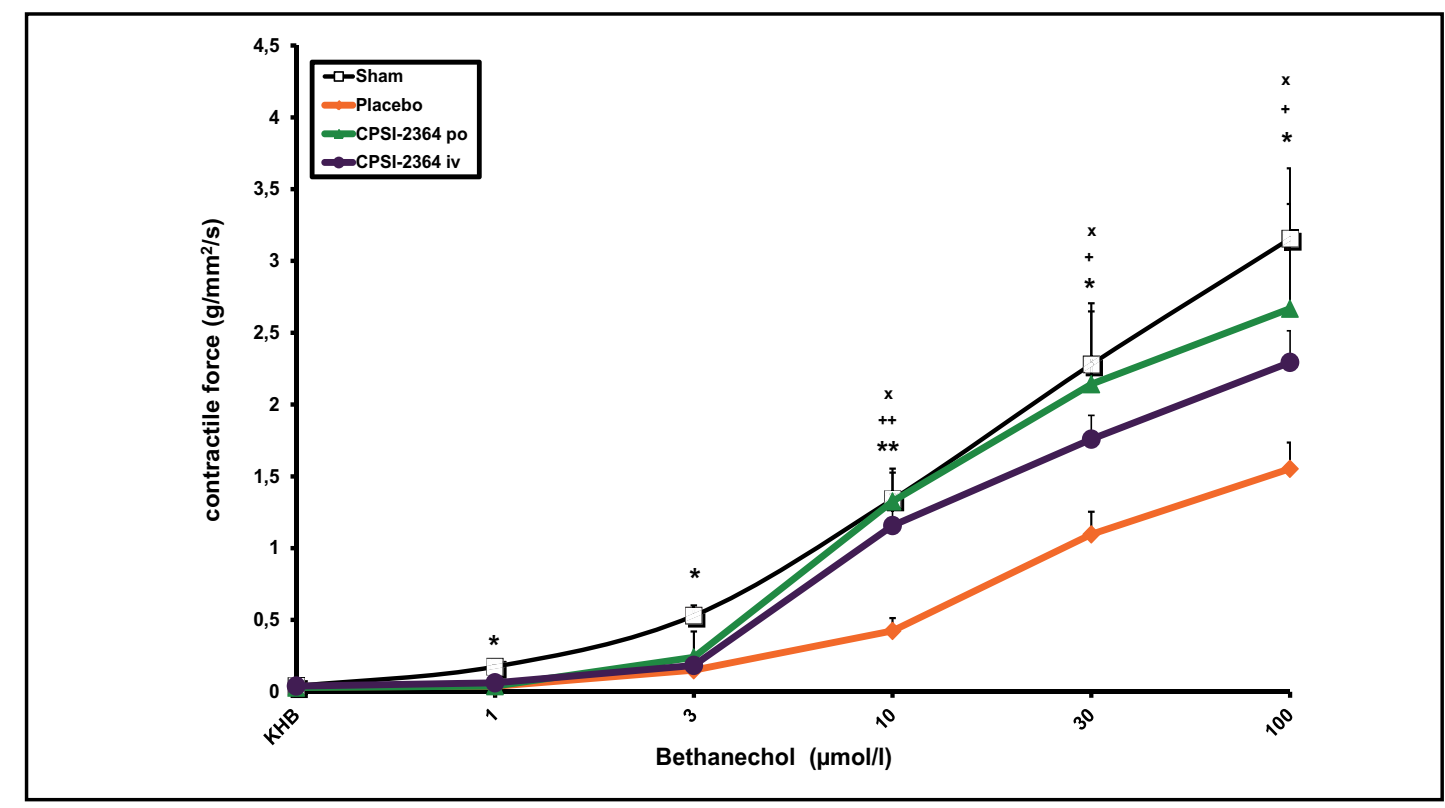

Fig. 2. In vitro measurement of spontaneous and bethanechol-induced contractility of ileal circular ME strips $24 \mathrm{~h}$ after operation. Bethanechol-induced contractility was significantly reduced in the IM+Placebo group at concentrations from $1 \mu \mathrm{M}$ to $100 \mu \mathrm{M}$ compared to sham operated animals. ME strips from CPSI2364-treated animals demonstrated an improved contractility after IM at $10 \mu \mathrm{M}$ to $100 \mu \mathrm{M}$ compared to IM+Placebo. Data are expressed as mean \pm SEM. Sham vs IM+Placebo: ${ }^{*} \mathrm{p}<0.05$ and ${ }^{* *} \mathrm{p}<0.01 ; \mathrm{IM}+\mathrm{CPSI}$ po vs. IM+Placebo; $+\mathrm{p}<0.05$ and $++\mathrm{p}<0.01$; IM + CPSI iv vs IM+Placebo $\times \mathrm{p}<0.05 . \mathrm{n}=4$ for sham and $n=6$ for IM groups.

$1.55 \pm 0.18$ vs. $3.16 \pm 0.73$ ). Spontaneous contractions did not differ between all groups. Oral CPSI-2364 treatment abrogated IM-induced contractility disturbances at $10 \mu \mathrm{M}(1.33 \pm 0.21$ $\left.\mathrm{g} / \mathrm{mm}^{2} / \mathrm{s}\right), 30 \mu \mathrm{M}(2.14 \pm 0.43)$ and $100 \mu \mathrm{M}(2.67 \pm 0.49)$ bethanechol. Intravenous application of CPSI-2364 led to an improved contractility compared to placebo treated swine at $10 \mu \mathrm{M}$ (1.16 \pm 0.22$), 30 \mu \mathrm{M}(1.76 \pm 0.17)$ and $100 \mu \mathrm{M}(2.29 \pm 0.22)$. Differences between orally or iv treated CPSI-2364 groups were not detected.

In vivo intestinal transit and colonic transit were analyzed simultaneously within the same swine via X-ray $24 \mathrm{~h}$ after surgery. In sham-operated placebo treated swine, all intestinal transit markers were distributed within the cecum and the proximal and middle colon (Fig. $3 A+E ;$ GC: $11.6 \pm 1.0$ ). IM showed a trend to delayed intestinal transit (GC: $7.7 \pm 4.3$ ) with most of the markers located within the distal small bowel (Fig. 3B+E) compared to

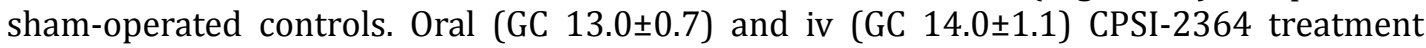
resulted in an enhanced GIT (Fig. 3C-E) compared to sham-operated and placebo-treated swine with most markers localized in the proximal colon. Clinical observation demonstrated a distended small bowel (which is a typical clinical sign for manifest POI) in placebo but not CPSI-2364 treated swine (Fig. $4 \mathrm{~A}+\mathrm{B}$, arrows). Distribution of the colonic markers (Fig. 3A-D; 3F) demonstrated no significant differences between the placebo-treated IM group (GC: $1.9 \pm 0.8$ ) and sham-operated controls (GC: $3.5 \pm 1.7$ ). Colonic transit in orally CPSI-2364 treated swine showed a trend to accelerated colonic transit (GC: $2.9 \pm 0.6$ ) while intravenous treatment resulted in significantly enhanced colonic transit time (GC 3.6 \pm 1.4 ).

\section{Anastomotic healing study}

Presence and function of macrophages is critical in anastomotic healing processes [16]. As we demonstrated before that CPSI-2364 affects postoperative macrophage cytokine and NO production in a rodent model of POI [14] we consequently analyzed intestinal 


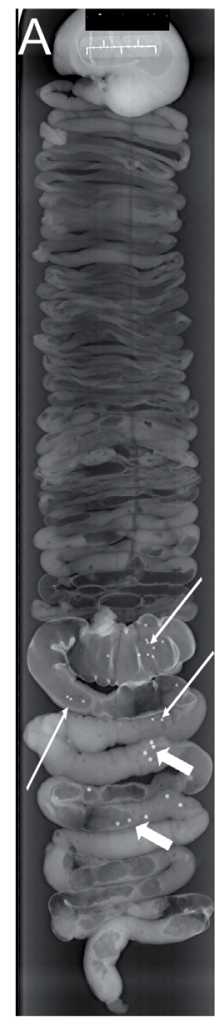

Sham

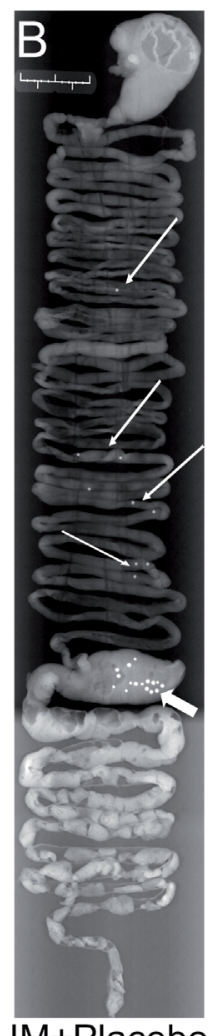

IM+Placebo

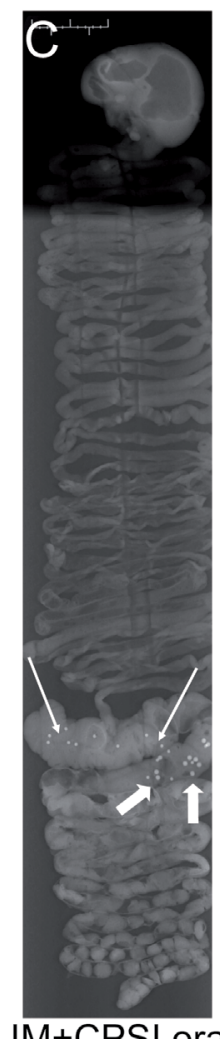

IM+CPSI oral

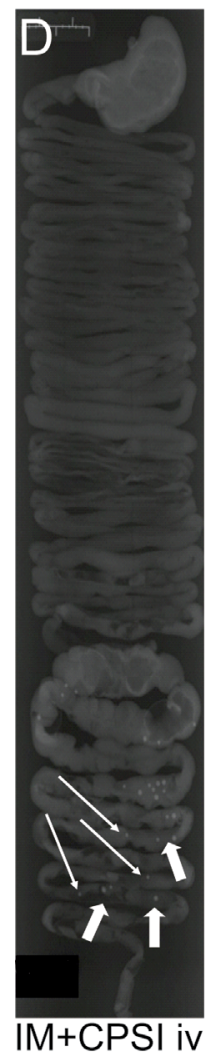

IM+CPSI iv
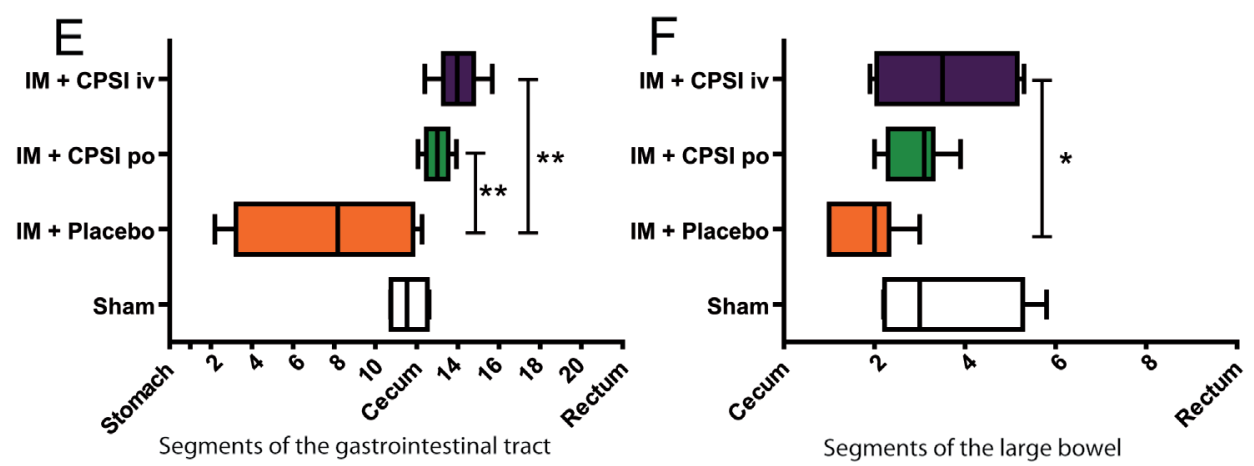

Fig. 3. Effects of CPSI-2364 on intestinal transit and colonic transit in swine. Swine underwent preoperative placebo or oral or iv treatment with CPSI-2364 before IM. At the end of IM, radio-opaque markers of different diameter were administered intraduodenally $(5 \mathrm{~mm}$, thin arrows) or into the cecum $(7 \mathrm{~mm}$, thick arrows) and X-ray analyses of the GI-tract was performed 24h after surgery. Representative X-ray analysis from (A) sham-operated animals and intestinally manipulated swine $24 \mathrm{~h}$ after (B) placebo or (C) oral or (D) intravenous CPSI2364 treatment. (E) Calculated geometric centers of intraduodenally applicated marker distribution along the GI tract. ${ }^{* *} \mathrm{p}<0.01$ vs. IM + Placebo (F) Calculated geometric centers of intracecally administered colonic transit markers. * $\mathrm{p}<0.05$ vs. IM + Placebo, $\mathrm{n}=4$ for sham, $\mathrm{n}=6$ for IM groups.

anastomotic healing after oral and iv administration of CPSI-2364 in swine. We decided to investigate anastomoses after a partial colon resection and rectorectostomy because leakage could be observed more often in the rectum than in the small bowel or colon. Additionally we considered postoperative day 6 for analyzes because anastomotic insufficiency is clinically often visible between postoperative days 5 to 7 . 
Fig. 4. CPSI-2364 prevents postoperative ileus in swine. Swine received preoperative placebo (A) or CPSI-2364 po or iv (B) and underwent IM procedure. After $24 \mathrm{~h}$ an abdominal inspection was performed. Pictures are representative images from $n=6$ animals per groups.
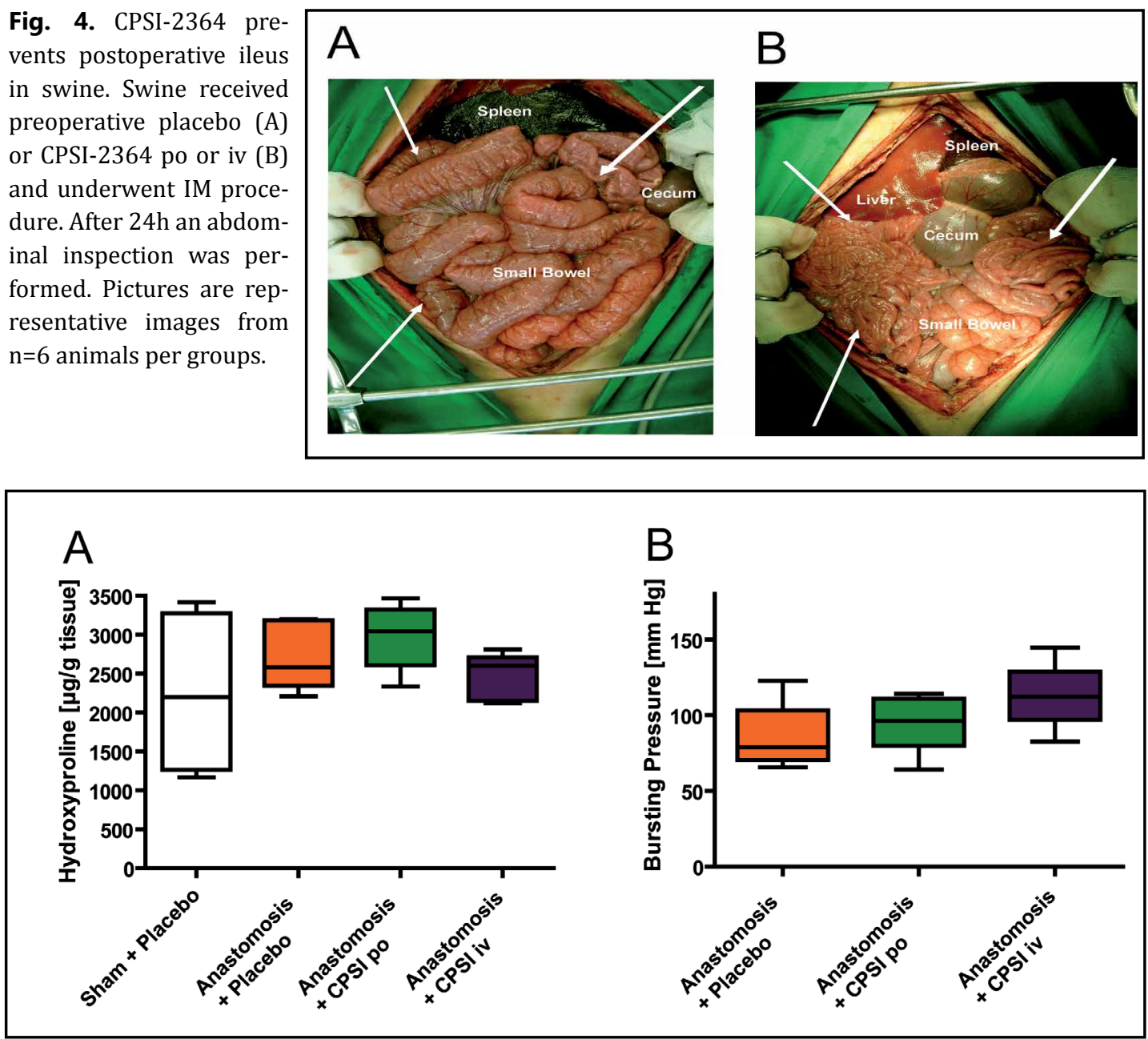

Fig. 5. Preoperative treatment with CPSI-2364 does not influence perianastomotic hydroxyproline concentration and anastomotic bursting pressure. Swine underwent partial colon resection and rectorectostomy after placebo or oral or iv CPSI2364 treatment. (A) Hydroxyproline concentration ( $\mathrm{n}=8$ per group) and (B) anastomotic bursting pressure ( $n=8$ per group) were analyzed 6 days after surgery. $n=8$ per group.

\section{Clinical observation and intraoperative examination}

All pigs completed the study with no major signs of illness. On postoperative day 6 swine underwent a relaparotomy. The abdominal wall, the abdominal cavity and the rectorectostomy were examined for wound healing disturbances (abscess formation, peritonitis or anastomotic leakage). Ultimately, none of the pigs showed any abnormalities in healing of the abdominal wall or the anastomoses, no adhesions were observed.

\section{Anastomotic healing}

It is well known that unaltered anastomotic healing strongly depends on regular macrophage function. As we could describe before CPSI-2364 temporarily suppresses macrophage activity in the small bowel ME. Therefore we analyzed submucosal collagen content which is crucial for anastomotic strength [23] by hydroxyproline measurement. HPC did not differ between sham-operated animals and operated swine treated with placebo $(2794 \pm 529 \mu \mathrm{g} / \mathrm{g}$ tissue), oral $(2981 \pm 396 \mu \mathrm{g} / \mathrm{g}$ tissue $)$ or intravenous CPSI-2364 (2518 \pm 276 $\mu \mathrm{g} / \mathrm{g}$ tissue) (Fig. 5A).

Additionally, mechanical strength of anastomoses (measured as ABP) was analyzed $e x$ vivo. ABP was indicated as a sudden loss of pressure and defined as the maximum intraluminal 
Fig. 6. Intravenous CPSI-2364 treatment impairs anastomotic healing. (A) Histopathological changes of the anastomotic region were graded 6 days after partial colon resection and rectorectostomy in CPSI2364- and placebo-treated swine ${ }^{* *} \mathrm{p}<0.01, \mathrm{n}=8$ per group). (B-D). Representative HE sections of paraffin - embedded swine rectorectostomies on postoperative day 6. Swine underwent preoperative (B) placebo, (C) CPSI-2364 per os or (D) CPSI-2364 iv. $\mathrm{Mu}$ : Mucosa, M: Muscularis, Gr: granulation tissue, R: re-epithelisation zone.

pressure prior to leakage. Preoperative oral or iv administration of CPSI-2364 did not impair ABP compared to placebo-treated swine (Fig. 5B: Placebo: $86 \pm 20 \mathrm{mmHg}$, CPSI 2364 oral: $94 \pm 18$, CPSI 2364 iv: $113 \pm 22$ ).

Finally, histopathological analyses were performed to determine whether CPSI2364 treatment affects anastomotic wound healing (Fig. 6A). On postoperative day 6 no differences were observed between the healing scores of oral CPSI-2364 (13.9 \pm 2.5$)$ or placebo $(13.7 \pm 2.6)$ treatment. However, a delayed healing with incomplete reepithelisation of the mucosa was observed within the intravenously treated CPSI-2364 group (Fig. 6B-D) leading to a significantly reduced healing score $(9.5 \pm 2)$.

Complications after CPSI-2364 treatment

In the oral CPSI-2364 treated group no adverse events could be observed. After intravenous administration of CPSI-2354 an anaphylactic shock with rash, hypotension and bronchospasm occurred once. Using iv application of corticosteroids and histaminereceptor blockers the symptoms disappeared rapidly.

Laboratory parameters (hemogram, electrolytes, liver and pancreatic enzymes, kidney values, coagulation factors) were checked at postoperative time points $(6 \mathrm{~h}, 24 \mathrm{~h}$, postoperative day 2,4 and 6) without any conspicuities (data not shown).

\section{Discussion}

It is widely accepted that POI originates from a post-traumatic inflammation of the ME, initiated by surgical manipulation of the intestine $[5-8,18]$. Currently, there is no prevention or evidence-based treatment of postoperative motility disorders. In the present swine study we demonstrate that the tetravalent guanylhydrazone CPSI-2364 prevents postoperative ME inflammation and subsequent POI after preoperative intravenous and oral application. Furthermore, we provide several lines of evidence that oral CPSI-2364 does not disturb
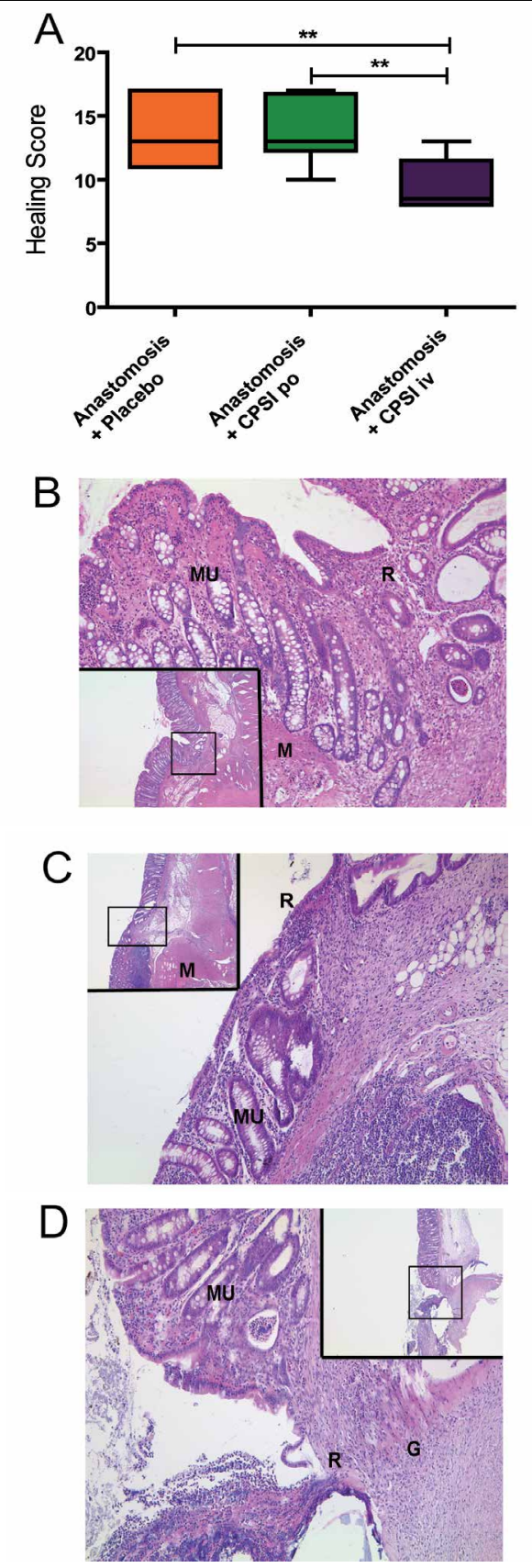
anastomotic wound healing after a rectorectostomy facing it out as a promising and safe option for prophylaxis of POI also in humans.

The clinically relevant prolonged form of POI originates from an inflammatory response within the ME induced by the surgical handling of the GI - tract during abdominal operation. Resident macrophages of the ME [10] have been shown to be the cellular key players in the onset of this inflammation. Once the local inflammation is induced it can spread along the entire GI tract [24] resulting in a panenteric dysmotility. In previous studies we have shown that elimination of the resident ME macrophages prevents POI in rodents [10]. As macrophage elimination is clinically contraindicated we investigated if a macrophage specific temporarily inactivation also prevents POI in rodents. Successfully, the tetravalent guanylhydrazone semapimod was identified to be effective in POI prevention. In this former study we used a chloride salt of semapimod, known as CNI-1493 which effectively prevented POI in mice partially via inactivation of peripheral macrophages and abrogation of macrophage-derived NO production [11]. Intravenous CNI-1493 was shown to reach brain levels high enough to centrally activate vagus nerve signaling which is known to fulfill antiinflammatory response in peripheral tissues, like the peritoneal cavity [25] and the GI tract [11]. The et al. further demonstrated that intracerebroventricular application of CNI-1493 also ameliorates POI [26] confirming its central mode of action next to the peripheral macrophage-inhibiting capacity. Importantly, effectiveness in POI prevention does not depend on simultaneous action of both, central vagus activating and peripheral macrophage inhibition, because intravenous application of CNI-1493 also prevents POI in vagotomized mice [14]. Although intravenous CNI-1493 was effective in POI prevention, systemic distribution and inactivation of resident macrophage population in other organs could be critical in a clinical setting.

CPSI-2364 is a mesylate salt form that, in contrast to CNI-1493, demonstrates oral bioactivity. In a previous study we demonstrated that oral CPSI-2364 prevents POI in mice. Importantly, oral administration leads to a near inclusive enrichment within the gastrointestinal wall and systemic distribution was not observed [14]. This indicates a lower risk of CPSI-2364 induced side effects. However, there is growing awareness that a disparity between mouse models of inflammatory diseases and their human counterparts exist [27]. Therefore and with regard to a future clinical use, we tested our previous findings in the present large animal model. We chose swine because of its similarity in wound healing processes and POI development compared to human [19, 28, 29]. Indeed, oral CPSI-2364 reduced postoperative inflammation in swine. This was shown by transcript reduction of the chemokine CCL2 that is crucial for leukocyte infiltration into the ME during POI [10] and a decrease of MPO activity (as a marker of PMN migration into the ME) after CPSI-2364 treatment. This reduction coincides with the improvement of smooth muscle contractility and an acceleration of intestinal transit.

Previous rodent studies demonstrated that surgical manipulation of the small bowel leads to dissemination of the inflammation to unmanipulated part of GI-tract followed by decreased motility. [24] Interestingly, the transit time of the unmanipulated colon shows a clear trend but no significant reduction in manipulated swine compared to sham operated animals. The missing statistical significance is probably based on both, a distinct degree of inflammation in sham-operated animals (who also received a laparotomy with two enterotomies for transit marker application) and the smaller group size in the shamoperated group. Nevertheless, intravenous CPSI-2364 application demonstrated significant improvement of colonic transit, indicating that CSPI-2364 also prevents dissemination of the ME inflammation. Subsumed, our former rodent and the present swine data revealed the preoperative prevention of macrophage activation by CPSI-2364 as a promising strategy for POI prophylaxis.

However, it is well known that immunocytes, particularly macrophages, facilitate wound healing processes in the GI-tract [16, 30,31] and express many mediators like NO [15] and cytokines [32] which are important for the anastomotic repair process. Wound healing disturbances may lead to leakage of intestinal anastomoses resulting in high perioperative 
morbidity and mortality. As CPSI-2364 acts via reduction of macrophage p38-MAPKdependent cytokine and NO liberation, we analyzed its effect on anastomotic wound healing in a rectorectostomy swine model. We chose anastomoses of the rectum because a higher incidence of leakages can be observed after rectorectostomy than in small bowel or colonic anastomoses. Although hydroxyproline concentration, a component of collagen that in turn mediates tensile strength of the anastomosis [23] and ABP did not differ among application route and presence of CPSI-2364, scoring of paraffin-embedded anastomoses linked the intravenous application with a significant reduced anastomotic healing. However, neither wound healing disturbances nor abscess formation were observed during relaparotomy. The delayed wound healing in the iv group may be explained by the systemic distribution of CPSI-2364 leading to an inactivation of circulating monocytes/macrophages that extravasate into the anastomosis and beneficially affect the healing process. As oral CPSI-2364 is nearly exclusively found within the GI wall, but not in the circulation, inactivation of circulating monocytes/macrophages is prevented [14].

In conclusion, this study demonstrates that preoperative oral and intravenous application of CPSI-2364 prevents POI in swine. Intravenous, but not oral application led to a delayed anastomotic healing after rectorectostomy. These findings point out oral CPSI-2364 treatment as a safe and promising strategy in POI prophylaxis.

\section{Abbreviations}

ME (muscularis externa); KHB (krebs-henseleit-buffer); GI (gastrointestinal); POI (postoperative ileus); PMN (polymorphonuclear neutrophils); p38-MAPK (p38 mitogen activated protein kinase); NO (nitric oxide); IM (intestinal manipulation).

\section{Acknowledgements}

We thank Mario Sitzia and Andrea Lohmer for assistance during swine operations and Ann-Christin Seelenbinder for her support performing radiography.

This study was supported by grants from the Deutsche Forschungsgemeinschaft (KA1270/3-1/2) and BONFOR (0-112.0040 and 0-112.0043).

\section{References}

1 Bauer AJ, Schwarz NT, Moore BA, Turler A, Kalff JC: Ileus in critical illness: mechanisms and management. Curr Opin Crit Care 2002;8:152-157.

- Iyer S, Saunders WB, Stemkowski S: Economic burden of postoperative ileus associated with colectomy in the United States. J Manag Care Pharm 2009;15:485-494.

- 3 Fukuda H, Tsuchida D, Koda K, Miyazaki M, Pappas TN, Takahashi T: Inhibition of sympathetic pathways restores postoperative ileus in the upper and lower gastrointestinal tract. J Gastroenterol Hepatol 2007;22:1293-1299.

-4 Bauer AJ, Boeckxstaens GE: Mechanisms of postoperative ileus. Neurogastroenterol Motil 2004;16 Suppl 2:54-60.

5 Kalff JC, Carlos TM, Schraut WH, Billiar TR, Simmons RL, Bauer AJ: Surgically induced leukocytic infiltrates within the rat intestinal muscularis mediate postoperative ileus. Gastroenterology 1999;117:378-387.

6 Kalff JC, Turler A, Schwarz NT, Schraut WH, Lee KK, Tweardy DJ, Billiar TR, Simmons RL, Bauer AJ: Intraabdominal activation of a local inflammatory response within the human muscularis externa during laparotomy. Ann Surg 2003;237:301-315.

7 Wehner S, Schwarz NT, Hundsdoerfer R, Hierholzer C, Tweardy DJ, Billiar TR, Bauer AJ, Kalff JC: Induction of IL-6 within the rodent intestinal muscularis after intestinal surgical stress. Surgery 2005;137:436-446.

-8 Kalff JC, Buchholz BM, Eskandari MK, Hierholzer C, Schraut WH, Simmons RL, Bauer AJ: Biphasic response to gut manipulation and temporal correlation of cellular infiltrates and muscle dysfunction in rat. Surgery 1999;126:498-509.

-9 Kalff JC, Schraut WH, Billiar TR, Simmons RL, Bauer AJ: Role of inducible nitric oxide synthase in postoperative intestinal smooth muscle dysfunction in rodents. Gastroenterology 2000;118:316-327. 


\section{Cellular Physiology $\quad$ Cell Physiol Biochem 2013;32:1362-1373 and Biochemistry \\ Vilz et al.: CPSI-2364 Prevents Postoperative Ileus in Swine}

10 Wehner S, Behrendt FF, Lyutenski BN, Lysson M, Bauer AJ, Hirner A, Kalff JC: Inhibition of macrophage function prevents intestinal inflammation and postoperative ileus in rodents. Gut 2007;56:176-185.

11 Wehner S, Straesser S, Vilz TO, Pantelis D, Sielecki T, de la Cruz VF, Hirner A, Kalff JC: Inhibition of p38 mitogen-activated protein kinase pathway as prophylaxis of postoperative ileus in mice. Gastroenterology 2009;136:619-629.

12 Wang H, Zhang M, Bianchi M, Sherry B, Sama A, Tracey KJ: Fetuin (alpha2-HS-glycoprotein) opsonizes cationic macrophagedeactivating molecules. Proc Natl Acad Sci U S A 1998;95:14429-14434.

13 Bianchi M, Ulrich P, Bloom O, Meistrell M, III, Zimmerman GA, Schmidtmayerova H, Bukrinsky M, Donnelley T, Bucala R, Sherry B, et al.: An inhibitor of macrophage arginine transport and nitric oxide production (CNI-1493) prevents acute inflammation and endotoxin lethality. Mol Med 1995;1:254-266.

14 Wehner S, Vilz TO, Sommer N, Sielecki T, Hong GS, Lysson M, Stoffels B, Pantelis D, Kalff JC: The novel orally active guanylhydrazone CPSI-2364 prevents postoperative ileus in mice independently of antiinflammatory vagus nerve signaling. Langenbecks Arch Surg 2012;;397:1139-47

15 Efron DT, Most D, Shi HP, Tantry US, Barbul A: Modulation of growth factor and cytokine expression by nitric oxide during rat colon anastomotic healing. J Gastrointest Surg 2003;7:393-399.

16 Pantelis D, Beissel A, Kahl P, Vilz TO, Stoffels B, Wehner S, Kalff JC: Colonic anastomotic healing in the context of altered macrophage function and endotoxemia. Int J Colorectal Dis 2011;26:737-746.

17 Bradley PP, Christensen RD, Rothstein G: Cellular and extracellular myeloperoxidase in pyogenic inflammation. Blood 1982;60:618-622.

18 Kalff JC, Schraut WH, Simmons RL, Bauer AJ: Surgical manipulation of the gut elicits an intestinal muscularis inflammatory response resulting in postsurgical ileus. Ann Surg 1998;228:652-663.

19 Moore BA, Overhaus M, Whitcomb J, Ifedigbo E, Choi AM, Otterbein LE, Bauer AJ: Brief inhalation of lowdose carbon monoxide protects rodents and swine from postoperative ileus. Crit Care Med 2005;33:13171326.

20 Jamall IS, Finelli VN, Que Hee SS: A simple method to determine nanogram levels of 4-hydroxyproline in biological tissues. Anal Biochem 1981;112:70-75.

21 Pantelis D, Beissel A, Kahl P, Wehner S, Vilz TO, Kalff JC: The effect of sealing with a fixed combination of collagen matrix-bound coagulation factors on the healing of colonic anastomoses in experimental high-risk mice models. Langenbecks Arch Surg 2010;395:1039-1048.

22 Biert J, Seifert WF, Verhofstad AA, Wobbes T, de Man BM, Hoogenhout J, Hendriks T: A semiquantitative histological analysis of repair of anastomoses in the rat colon after combined preoperative irradiation and local hyperthermia. Radiat Res 1998;149:372-377.

23 de Hingh IH, van Goor H, de Man BM, Lomme RM, Bleichrodt RP, Hendriks T: Selective cyclo-oxygenase 2 inhibition affects ileal but not colonic anastomotic healing in the early postoperative period. Br J Surg 2006;93:489-497.

-24 Schwarz NT, Kalff JC, Turler A, Speidel N, Grandis JR, Billiar TR, Bauer AJ: Selective jejunal manipulation causes postoperative pan-enteric inflammation and dysmotility. Gastroenterology 2004;126:159-169.

25 D'Souza MJ, Oettinger CW, Milton GV, Tracey KJ: Prevention of lethality and suppression of proinflammatory cytokines in experimental septic shock by microencapsulated CNI-1493. J Interferon Cytokine Res 1999;19:1125-1133.

26 The F, Cailotto C, van d, V, de Jonge WJ, Bennink RJ, Buijs RM, Boeckxstaens GE: Central activation of the cholinergic anti-inflammatory pathway reduces surgical inflammation in experimental post-operative ileus. Br J Pharmacol 2011;163:1007-1016.

27 Of men, not mice. Nat Med 2013;19:379.

-28 Walters EM, Prather RS: Advancing swine models for human health and diseases. Mo Med 2013;110:212215.

29 Hoeppner J, Crnogorac V, Hopt UT, Weiser HF: The pig as an experimental model for colonic healing study of leakage and ischemia in colonic anastomosis. J Invest Surg 2009;22:281-285.

-30 Pull SL, Doherty JM, Mills JC, Gordon JI, Stappenbeck TS: Activated macrophages are an adaptive element of the colonic epithelial progenitor niche necessary for regenerative responses to injury. Proc Natl Acad Sci U S A 2005;102:99-104.

-31 Compton R, Williams D, Browder W: The beneficial effect of enhanced macrophage function on the healing of bowel anastomoses. Am Surg 1996;62:14-18.

32 Rappolee DA, Mark D, Banda MJ, Werb Z: Wound macrophages express TGF-alpha and other growth factors in vivo: analysis by mRNA phenotyping. Science 1988;241:708-712. 